\title{
Example-Based Machine Translation in the Pangloss System
}

\author{
Ralf D. Brown \\ Center for Machine Translation \\ Carnegie Mellon University \\ 5000 Porbes $\Lambda$ venue \\ P'ittisburgh, PA 15213-3890 \\ ralf@cs.cmu , edu
}

\begin{abstract}
The Pangloss Example- Based Machine Translation engine (PanEBMT)' is a translation system requiring essentially no knowledge of the structure of a language, merely a large parallel corpus of example sentences and a bilingual dictionary. Input texts are segmented into sequences of words occurring in the corpus, for which translations are deternined by subsentential alignment of the sentence pairs containing those sequences. These partial translations are then combined with the results of other translation engines to form the final translation produced by the Pangloss system. In an internal evaluation, PanEBMT achicved $70.2 \%$ coverage of unrestricted Spanish news-wire text, despite a simplistic subscnticntial alignment algorithm, a suboptimal dictionary, and a corpus from a different domain than the evaluation texts.
\end{abstract}

\section{Introduction}

Pangloss (Nirenburg et al., 1995) is a multiengine machine translation system, in which scvcral translation engines are run in parallel to propose translations of various portions of the input, from which the final iranslation is selected by a statistical language model. PanlBMT is one of the tramslation engines used by Pangloss.

LBM'l is essentially translation-by-analogy: given a source-language passage $S$ and a collection of aligned source/target text pairs, find the "best" match for $S$ in the sourcc-language half of the text collection, and accept the target-language half of that match as the translation. PanEBMT', like other example-based translation systems, uses essentially no knowledge about its source or target languages; what little knowledge it docs use is optional, and is supplied in a configuration file. It s

\footnotetext{
'This work as part of the l'angloss project was supported by the U.S. Department of Defense
}

three main knowledge sources are: a sententiallyaligned parallel bilingual corpus; a bilingual dictionary; and a target-language root/synonym list. The fourth (minor and optional) knowledge source is the language-specific information provided in 1.he configuration file, which consists of a list of tokenizations equating words within classes such as werkelays, a list of words which may be elided during alignment (such as articles), and a list of words which may be inserted

\section{Parallel Bilingual Corpus}

'The corpus used by Pan EBM'T' consists of a set of source/target sentence pairs, and is fully indexed on the source-language sentences. 'The corpus is not aligned at any granularity finer than the sentence pair; subsentential alignment is performed at run-time based on the sentence fragments selected and the other knowledge sources.

The corpus index lists all occurrences of cvery word and punctuation mark in the sourcelanguage sentences contained in the corpus. The index has been designed to permit incremental updates, allowing new sentence pairs to be added to the corpus as they become available (for example, to implement a translation memory with the systern's own output). 'The text is tokenized prior to indexing, so that words in any of the equivalence classes defined in the EBMT configuration file (such as month names, countries, or mcasuring units), as well as the predefined equivalence class <number>, are indexed under the equivalence. class rather than their own narres. For each distinct token, the index contains a list of the token's occurrences, consisting of a sentence identifier and the word number within the sentence. $\Lambda t$ translation time, PanEBMT back-substitutes the appropriate target-language word into any translation which involves any tokenized words.

'The bilingual corpus used for the results roported here consists of 726,406 Spanish-Linglish sentence pairs drawn primarily from the UN Multilingual (orpus available from the Linguistic D)ata Consortium (Graff and Finch, 1992) (Figure 1), with a small admixture of texts from the Pan- 


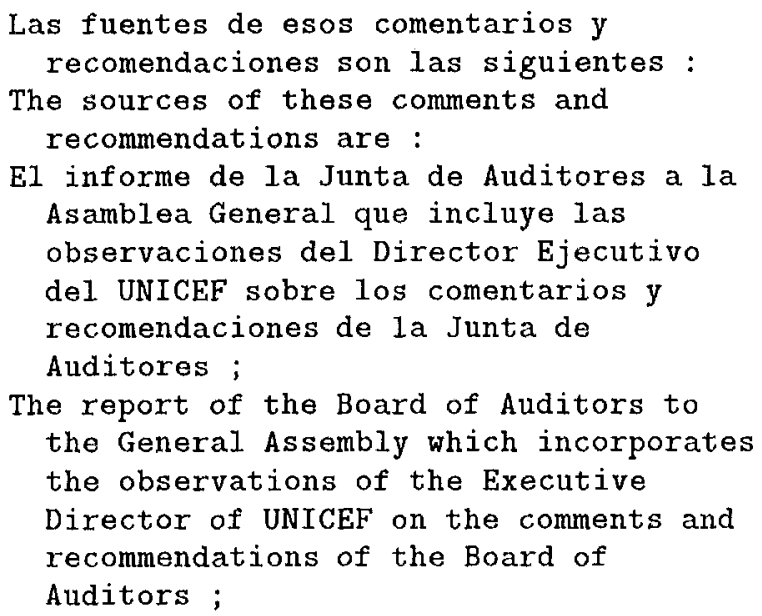

Figure 1: Corpus Sentence Pairs

American Health Organization and prior project evaluations ${ }^{2}$, indexed as described above.

Together, the bilingual dictionary and targetlanguage list of roots and synonyms (extracted from WordNet when translating into English) provide the necessary information to find associations between source-language and targetlanguage words in the selected sentence pairs. These associations are used in performing subsentential alignment. $\Lambda$ source word is considered to be associated with a target-language word whenever either the target word itsclf or any of the words in its root/synonym list appear in the list of possible translations for the source word given by the dictionary.

Not all words will be associated one-to-one; however, the current implementation requires that at lcast one such unique association be found in order to provide an anchor for the alignment process.

\section{Implementation}

PanEBMT is implemented in $\mathrm{C}++$, using the PramepaC library (Brown, 1996) for accessing Lisp data structures stored in files or sent from the main Pangloss module via Unix pipes. PanlEBMT consists of approximately 13,300 lines of code, including the code for a glossary mode which will not be described here.

PanEBMT uses a re-processed version of the bilingual dictionary used by Pangloss's dictionary translation engine (Figure 2). The re-processing consists of removing various high-frequency words and splitting all multi-word definitions into a list of single words, needed to find one-to-one associations.

\footnotetext{
${ }^{2} 10250$ sentence pairs stem from the PAIO corpus and 552 pairs from evaluations.
}

\author{
(ACADMICOS ACADEMICS ACADEMICAL \\ TITLES DEGREES) \\ (ACAECIDO HAPPEN) \\ (ACAECIDOS HAPPEN) \\ (ACANTONADAS CANTON QUARTER TROOPS) \\ (ACANTONAMIENTO CANTONMENT) \\ (ACARREA CARRY CART HAUL TRANSPORT \\ CAUSE OCCASION) \\ (ACARREABA CARRY CART HAUL TRANSPORT \\ CAUSE OCCASION) \\ ... \\ (ACARREARON CARRY CART HAUL TRANSPORT \\ CAUSE OCCASION) \\ (ACARREAR TRANSPORT HAUL CART CARRY \\ LUG ALONG BRING DOWN CAUSE OCCASION \\ ITS TRAIN RESULT GIVE RISE)
}

Figure 2: Bilingual Dictionary Entries

\section{EBMT's Place in Pangloss}

PanEBMT is merely onc of the translation engines used by Pangloss; the others are transfer engines (dictionaries and glossaries) and a knowledge-based machine translation engine (Figure 3). Each of these produces a set of candidate translations for various segments of the input, which are then combined into a chart (Figure $3)$. The chart is passed through a statistical language model to determine the best path through the chart, which is then output as the translation of the original input sentence.

\section{EBMT Operation}

The EBMT engine produces translations in two phases:

1. find chunks by searching the corpus index for occurrences of consecutive words from the input text

2. perform subsentential alignment on each sentence pair found in the first phase to determine the translation of the chunk

In constrast with other work on examplebased translation, such as (Maruyama and Watanabe, 1992) or early Pangloss EBMT experiments (Nirenburg et al., 1993), PanEBMT does not find an optimal partitioning of the input. Instead, it attempts to produce translations of every word sequence in the input sentence which appears in its corpus. The final selection of the "correct" cover for the input is left for the statistical language model, as is the case for all of the other translation engines in Pangloss. An advantage of this approach is that it avoids discarding possible chunks merely because they are not part of the "optimal" cover for the input, instead selecting the input coverage by how well the translations fit together to form a complete translation. 


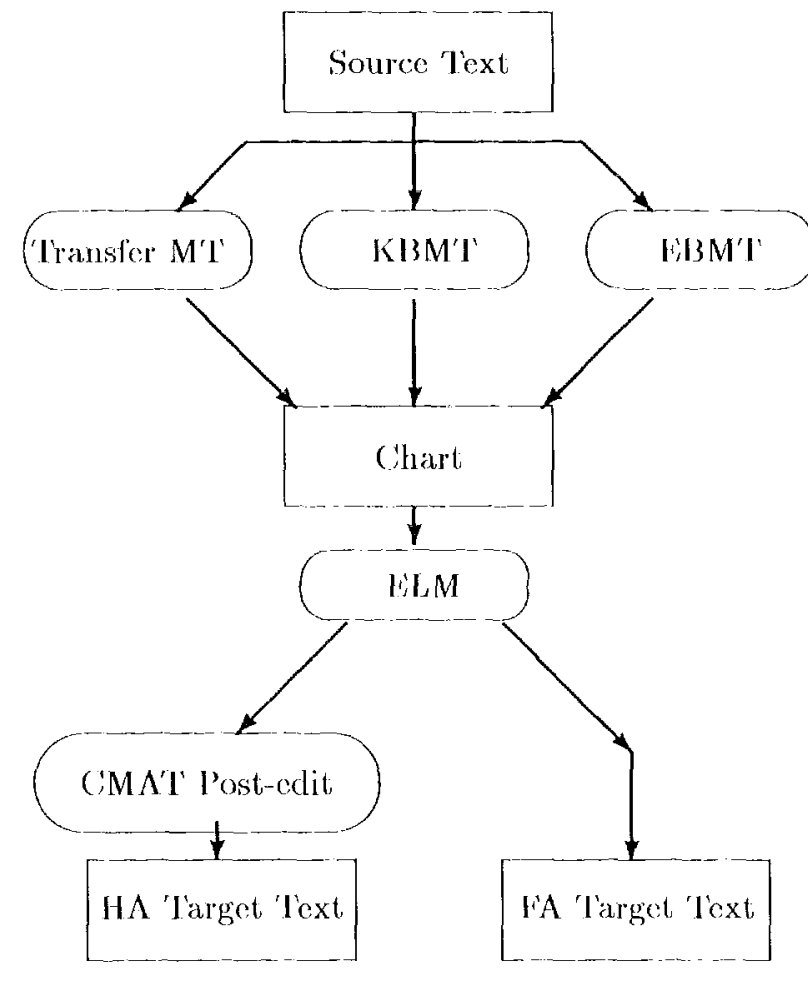

ligure 3: Pangloss Machine Translation System Architecture

'To find chunks, the engine sequentially looks up each word of the input in the index. 'The occurrence list for each word is compared against the occurrence list for the prior word and against the list of chunks extending to the prior word. For each occurrence which is adjacent to an occurrence of the prior word, a new chunk is created or an existing chunk is cxtended as appropriate. After processing all input words in this manner, the engine has determined all possible substrings of the input containing at least two words which are present in the corpus. Since the more frequent word sequences can occur hundreds of tirnes in the corpus, the list of chunks is culled to eliminate all but the last five (by default) occurrences of any distinct word sequence. By selecting the last occurrences of each word sequence, one effectively gives the most recent additions to the corpus the highest weight, precisely what is needed for a translation memory.

Next, the sentence pairs containing the chunks found in the first phase are read from disk, and alignment is performed on each in order to determine the translation of the chunk--unless the match is against the entire corpus entry, in which case the entire target-language sentence is taken as the translation. Alignment currently uses a rather simplistic brute-force approach-very similar to that of (Nircnburg, et al., 1994)-which iden- tifies the minimum and maximmm possible segments of the target-language sentence which could possibly correspond to the chunk, and then applics a scoring function to every possible substring of the maximum segment containing at least the minimum segment. 'The substring with the best score is then selected as the aligned match for the chunk.

'The aligument scoring function is computed from the weighted sum of a number of extremely simple test functions. The woights can be changed for differing lengths of the source chunk in order to adapt to varying impacts of the tests with varying numbers of words in the chunk, as well as vary. ing impacts as some or all of the raw test scorcs change. 'The test functions include (in approximate order of importance) such measures as a) the number of sourec words without correspondences in the target, b) the number of target words without correspondences in the source, $\mathbf{c}$ ) matehing words in source/target without correspondences, d) number of words with correspondence in the full target but not the candidate chunk, e) commou sentence boundaries, f) elidable source words, $g$ ) insertable target words, and h) the difference in length between sonrce and target chunks.

There is one exception to the above procedure for retrieving and aligning chunks. If any of the chunks covers the entire imput string and the entire source-language half of a corpus sentence pair, then all other chunks are discarded and the targetlanguage half of the pair is produced as the translation. This specds up the system when operating in translation menory mode, as would be the case in a system used to translate revisions of previous texts. Unlike a pure translation memory, however, l'anlisM'l' does not, require an exact match with a menorized translation.

Figure 4 shows the set of translations generated from one sentence. 'The output is shown in the format used for standalone testing, which gencrates only the best translation for each distinct chunk; when integrated with the rest of l'angloss, Panl'BM'I' also includes information indicating which portion of the input sentence and which pair from the corpus were used, and can produce multiple translations for each chunk. T'ho number next to the source-language chunk in the ontput indicates the value of the scoring function where higher values are worse. Very poor alignments (scores greater than five times the source chunk length) have already been omitted from the output.

\section{Recent Enhancements}

'Ihe I'BMA' engine described here is a completely now implementation in C ++ replacing an earlier Lisp version. The previous version had performed very poorly (to the point where its results were 


$\begin{array}{lll}\text { Input words } & & 9169 \\ \text { Matched against corpus } & 90.4 \% & 8294 \\ \text { Alignable } & 84.5 \% & 7748 \\ \text { Good alignments } & 70.2 \% & 6439\end{array}$

El Banco de Santander habia sido elegido el lunes por las autoridades monetarias espanolas para comprar el Banco Espanol de Credito (Banesto), cuarto banco espanol.

"El Banco de" (0)

("the Bank of")

"El Banco de Santander" (1)

("the Bank of Santander")

"Banco de" (0)

("Bank of")

"Banco de Santander" (1)

("Bank of Santander")

"de Santander" (0)

("of Santander")

"habia sido" (0.5)

("been")

"elegido el" (0)

("chosen the")

"el lunes por" (0)

("Monday by the")

"por las" (0)

("by the")

"por las autoridades" (14.2)

("by the health authorities")

"por las autoridades monetarias" (0)

("by the monetary authorities")

"las autoridades monetarias" ( 0 )

("the monetary authorities")

"comprar el" (0)

("buying the")

"Espanol de Credito" (13.2)

("Spanish Institute of Credit for")

"de Credito" (0)

("of credit")

"de Credito (" (1)

("of credit (")

"Credito (" (0)

("credit (")

", cuarto" (0)

(", fourth")

"banco espanol" (0)

("Spanish bank")

"espanol ." (0)

("Spanish.")

Figure 4: Sample Translations
Table 1: Coverage and Sentence Alignability

\begin{tabular}{lccccc} 
Engine & \multicolumn{2}{c}{ Proposed } & \multicolumn{2}{c}{ Selected } \\
Name & Arcs & Words & Arcs & Words & Cover \\
\hdashline DICT & 27482 & 27482 & 3451 & 3451 & 9167 \\
EBMT & 11005 & 34992 & 1527 & 4768 & 6439 \\
GLOSS & 17663 & 19249 & 1567 & 1774 & 5780 \\
\hdashline & & & \\
Overal1: & 46580 & 71998 & 5415 & 9169 & 9169
\end{tabular}

Table 2: Contributions of Pangloss Engines

essentially ignored when combining the outputs of the various translation engines), for two main reasons: inadequate corpus size and incomplete indexing.

The earlier incarnation had used a corpus of considerably less than 10 megabytes of text, compared to the 270 megabytes used for the results described herein. 'The seven-fold increase in corpus size produces a proportional increase in matches.

Not only was the corpus fairly small, the text which was used was not fully indexed. To limit the size of the index file, a long list of the most frequent words were omitted from the index, as were punctuation marks. Although allowances were rrade for the words on the stop list, the missing punctuation marks always forced a break in chunks, frequently limiting the size of chunks which could be found. Further, allowance was made for the un-indexed frequent words by permitting any sequence of froquent words between two indexed words, producing many erroncous matches.

The newer implementation fully indexes the corpus, and thus examines only exact matches with the input, ensuring that only good matches are actually processed. Further, PanEBMT' can index certain word pairs to, in effect, precompute some two-word chunks. When applied to the five to ten most frequent words, this pairing can reduce processing time during translation by dramatically reducing the amount of data which must bo read from the index file (for example, there might be 10,000 occurrences of a word pair instead of $1,000,000$ occurrences of one of the words and 100,000 of the other word), and thus the number of adjacency comparisons which must be made.

\section{Performance}

\subsection{Accuracy}

PanEBMT was first put to the test during an 
internal evaluation in August 1995, which was similar in design to the $\Lambda$ RI'A MT evaluations (White \& O' 'omell, 1994). During this cvaluation, twenty newswire articles (selected from the 100 articles used in the prior ARPA evaluation) averaging about 450 words each were processed and subsequently exantined. Vor this paper, another evaluation was performed using a subset of the Pangloss system on the 253.3 sentences in the twenty articles. 'Iable 2 shows the total number of ares proposed by each translation cngine used, the number selected for ontput by the stat tistical language model, and the number of souree words represented by those ares. 'The final colunrm shows the total number of souree words covered by at least one proposed are. 'T'he values for individual engines to not sum to the Overell value because multiple engines can produce equivalent ares, which are combince in the chard, with both engines credited for the are. 'The congines listed in the tables are

- DrCTionary: PanlibM'l's association diclionary, used here primarily to provide coverage for words not otherwise covererl

\section{- EBMT': Pan Elsmó}

- GLOSSaries: hand-crafted word/phrase bilingual glossaries

\subsection{Speed}

Indexing a 270 megaloyte corpus reculues approximately 45 minules on a Sum Sparestation LX when all files are located on local disks, and another 80 minntes to pack the index (not, required, but int proves speed at run tinc). Incremental adelition of new data to the corpus procecels at a rate of roughly six megrabytes per minute.

$\Lambda$ sample text of 15 sentences totalling $1 / 4$ words and punctuation marks can be processed in just under three minutes. 'The 20 texts used in the evaluation can bo completely processed in l.wo hours, inclurling separate passes for dictiomay lookups and statistical modeling by a separate progran (described in (Brown and lirederking, 1995)); P'anlisM'l' accounts for about 80 minutes of those two hours.

'I'he above timings represent a variety of ipeed optimizations which have been applied since the August 1995 evaluation, resulting in a coubling of the indexing speed and tripling of translation specel.

\section{Strengths and Weaknesses}

As currently implenented, Panblime has both strengths and weaknesses. Its strengths are that the minimal knowledge required allows quick retargeting and that, its design provides for graceful degratation. Its weaknesses are that it is umable to completely cover inputs, that it doess not perform well when the correspondenees between source-language and target-language words are not one-to-one, and that (like statisticallybased translation systems) it is sensitive to difforences between the example corpus and the sentences to be translated.

'The astute reader will have noticed that there have been virtually no mentions of the source or target languages in this paper-they are not relevant to discussions of the design and operation of the engine, since the only languagedependent knowledge consists of the equivalenes classess and the lists of insertable and clidable. words, which are provided via the configuration file. 'This language-independent aspect of LBM'T' tnakes PanlisM'I rapidly retargetable to other language pairs, and in fact there are already versions of Pantil3M'T providing Serbocroalian-tolinglish and linglish-to-Serbocroatian translations (no experimental data is as yet available for Serhocroatian because the complete dictionary and corpus are still being acculued). (iven the three required knowledge sourees of corpus, dictionary, and word-root list, P'an filBM'I' can begin produe:ing tanslations for a new language pair in only a fow hours. Iriue tuniug will require one to two weeks to determine reasonable word classes for tokenization (along with the required re-indexing of the corpus) and to adjust the scoring function weights:

Number and quality of transtations degrades gradually as the size and quality of the bilingual dietionary and synonym list decrease. An in-complete dietionary or root/symonym list merely causes P'ankBBM'l' to miss sone potential translattions. Similarly, a smaller corpus produces fower potential matches, but there is no point for any of the three knowledge sourees at which the engine suddenly ceases to function. One can take andvantage of this gradual behavior by building the knowledge sources increneutally and using LBB M'T' for transtations even before the knowledge sources have been compleded. In particular, by adding, post-reditod ontput of the M'L system back into the corpus, the system an be bootstrapped from a relatively modest initial corpus (precisely the idea behind a translation memory).

Juring preparation of this paper, several extrancous lines were discovered in the corpus files, which cansed more than 29,400 sentence pairs (over $4 \%$ of the corpus) to be corrupted. Due to the extra lines, the corrupted pairs consisted of the Pinglish target sentence from one pair and the Spantish source sentence from the following pair. 'This error had not been discovered earlier becaulse it had no obvious effect on PanlisM'l's performance a clear example of the system's graceful degradation property.

Lack of complete inpul coverage is a severe ob stacte to using l'anl'BM'l' as a stand-alone trans- 
lation system. The engine can not generate a chunk for a word unless it both co-occurs with either the preceding or following word somewhere in the corpus, and at least one occurrence can be successfully aligned. Additionally, candidate chunks are omitted if the alignment was successful but the scoring function indicates a poor match. Unless all of these conditions are met, a gap in output occurs for the particular input word. In the context of the Pangloss system, such gaps are not a problem, since one of the other engines can usually supply a translation covering each gap.

As currently implemented, the EBMT engine is unable to properly deal with translations that do not involve one-for-one correspondences between source and target words (e.g. Spanish "mil milliones" corresponding to English "billions"). Lack of a one-to-one correspondence between sourcelanguage and target-language expressions can often cause the alignment to be incorrect or fail altogether under the current alignment algorithm.

Since the corpus used in the experiments described here was based almost entirely on the UN proceedings rather than newswire text, PanEBMT did not find many long chunks during the evaluation. In fact, the average chunk was just over three words in length, and less than three percent of the chunks were more than six words long. This quite naturally affects the quality of the final translation, since many short pieces must be assembled into a translation rather than one or two long segments.

Despite all these difficulties, PanEBMT was able to cover $70.2 \%$ of the input it was presented with good chunks, and generate some translation for more than 84ordinarily not output at all). Integrating the hand-crafted glossaries from Pangloss into the corpus, thus adding 148,600 effectively pre-aligned phrases to the corpus, improved the matches against the corpus from $90.4 \%$ to $90.9 \%$ of the input, and the coverage with good chunks to $73.3 \%$.

\section{$9 \quad$ Future Enhancements}

Since PanEBMT is a fairly new implementation, there is still much that could be done to enhance it. Among the improvements being considered are: improving the quality of the dictionary (in progress); supporting one-to-many or manyto-one associations for alignment; optimizing the test-function weights; other alignment algorithms; using linguistic information such as morphological variants and source-language synonymy to increase the number of matches against the corpus; using approximate matchings when no exact matches exist in the corpus; and using of a classifier algorithm to remove redundancy from the corpus (suggested by C. Domashnev).

\section{References}

Ralf Brown (in preparation). FramepaC User's Manual Carnegie Mellon University Center for Machine Translation technical memoran$\mathrm{dum} h t t p: / / w w w . c s . c m u . e d u / a f s / c s . c m u . e d u /-$ user $/ \mathrm{ralf} /$ pub/WWW/papers.html

Ralf Brown and Robert Frederking 1995. Applying Statistical English Language Modeling to Symbolic Machine Translation. In Proceedings of the Sixth International Conference on Theoretical and Methodological Issues in Machine Translation (TMI-95), pages 221-239. Leuven, Belgium.

David Graff and Rebecca Finch 1994. Multilingual Text Resources at the Linguistic Data Consortium In Proceedings of the 1994 ARPA Human Language Technology Workshop Morgan Kaufmann.

H. Maruyama and H. Watanabe 1992. Tree Cover Search Algorithm for Example-Based Translation. In Proceedings of the Fourth International Conference on Theoretical and Methodological Issues in Machine Translation (TMI-92), pages 173-184. Montreal.

M. Nagao 1984. A Framework of a Mechanical Translation between Japanese and English by Analogy Principle. In Artificial and Human Intelligence, A. Elithorn and R. Banerji (eds). NATO Publications

Sergei Nirenburg, (ed.). 1995. "The Pangloss Mark III Machine Translation System." Joint Technical Report, Computing Research Laboratory (New Mexico State University), Center for Machine Translation (Carnegie Mellon University), Information Sciences Institute (University of Southern California). Issued as CMU technical report CMU-CMT-95-145.

Sergei Nirenburg, Stephen Beale, and Constantine Domashnev 1994. A Full-Text Experiment in Example-Based Machine Translation. In New Methods in Language Processing Manchester, England.

Sergei Nirenburg, Constantine Domashnev, and Dean J. Grannes 1993. Two Approaches to Matching in EBM'T'. In Proceedings of the Iifth International Conference on Theoretical and Methodological Issues in Machine Translation ('TMI-93).

White, J.S. and 'T. O'Connell. 1994. "Fvaluation in the ARPA Machine 'Translation Program: 1993 Methodology." In Proceedings of the ARPA HLT Workshop. Plainsboro, NJ. 